\title{
Ionic transport in the amorphous phase of semicrystalline polyethylene oxide thin films
}

\author{
Daniel E. Martínez-Tong ${ }^{1,2, *}$, Luis A. Miccio ${ }^{2, \dagger}$, Angel Alegria ${ }^{2,3, *}$ \\ ${ }^{1}$ Donostia International Physics Center. P. M. Lardizabal 4, 20018 Donostia, Spain. \\ ${ }^{2}$ Centro de Física de Materiales (CSIC-UPV/EHU). P. M. Lardizabal 5, 20018 Donostia, Spain. \\ ${ }^{3}$ Departamento de Física de Materiales (UPV/EHU), Apdo. 1072, 20080 Donostia, Spain. \\ ${ }^{\dagger}$ Present address: Bihurcrystal. P Mikeletegi 83, 20009, Donostia, Spain. \\ *corresponding authors: danielenrique_martineztong001@ehu.eus \\ angel.alegria@ehu.eus
}

\begin{abstract}
We present a detailed study on the ionic transport properties of polyethylene oxide (PEO) thin films prepared under different conditions. Using a state of the art Atomic Force Microscopy (AFM) methodology we acquired simultaneously the nanostructured topography of these semicrystalline polymer films as well as the corresponding dielectric function; in the latter case by probing the frequency dependent tip-sample electrical interactions. By means of this AFM protocol we studied the ionic conductivity in the PEO amorphous phase and its dependence with film preparation conditions. In general, for any preparation method we found a distribution of conductivities ranging from $10^{-14}$ to $10^{-6} \mathrm{~S} / \mathrm{cm}$. Specifically, PEO thin films crystallized from the melt presented relatively high conductivity values, which decreased in PEO films prepared from solutions at room temperature depending on solvent polarity. We discuss our results by considering the molecular arrangement of the polymer segments in the complex amorphous phase, which is strongly influenced by the PEO crystallization route.
\end{abstract}

Keywords: Ionic transport, Broadband Dielectric Spectroscopy, Atomic Force Microscopy, Polymer electrolytes, Semicrystalline Polyethylene oxide 


\section{Introduction}

Polymer electrolytes consist of a solid polymer matrix doped with lithium salts, where ions can move in the space provided by the free volume of the matrix and conductivity is thus possible above the glass transition temperature $\left(T_{\mathrm{g}}\right)$ of the polymer, i.e. in the state where the polymer chains in the amorphous phase are highly mobile. There, ion transport is influenced by the motion of polymer segments repeatedly creating new coordination sites into which the ions migrate. ${ }^{1-4}$ Among several candidates, polyethylene oxide (PEO) has emerged as one of the most commonly used polymer matrices ${ }^{3-7}$ and can be considered as a model system. For high enough molecular weight (above $\sim 1000 \mathrm{Da}$ ) this polyether is a solid material at room temperature, although its $T_{\mathrm{g}}$ is about $210 \mathrm{~K}$. The PEO room temperature mechanical stability is provided by its semicrystalline nature; however, the polymer crystalline phase affects strongly the overall behavior, since the presence of PEO crystals reduces the amount of amorphous material, leading to changes in the final molecular structure and reducing the polymer conductivity due to the appearance of hindrances altering ion motion. ${ }^{3,8,9}$

In semicrystalline polymers, the molecular ordering and packing efficiency in the amorphous phase is not only affected by the amount of crystalline domains but also on the details of the crystallization process. ${ }^{10,11}$ In this way, the resulting amorphous phase is not homogeneous, but a compendium of fractions: ${ }^{10,12,13}$ each one of them differently affected by the surrounding crystals. Moreover, when working with nanometric systems as thin films, crystallization is also influenced by factors as substrate and solvent nature, ${ }^{14,15}$ post-treatment protocols ${ }^{16}$ and possible formation of adsorbed layers. ${ }^{17,18}$ Specifically, in the preparation of PEO thin films solvent polarity has a key role in the resulting crystalline structure, ${ }^{15}$ due to the complex interplay between processing conditions, molecular mass, and solubility. Also, the formation of an adsorbed layer in PEO thin films acts on the melt crystallization and dewetting behavior. ${ }^{16}$ Considering the increasing use of thin film polymer electrolytes, ${ }^{7,} 19-21$ it is of great interest to address how the crystallization process of PEO thin films influences the ionic transport properties. This effect would be related not only to the film crystallinity but more importantly by the impact of the crystalline phase on molecular packing in the remaining amorphous material.

In this work we characterize the ionic conductivity in the amorphous phase of $200 \mathrm{~nm}$ PEO semicrystalline thin films by using nanoDielectric Spectroscopy (nDS), ${ }^{22-25}$ a non-contact Atomic Force Microscopy (AFM) technique that probes the frequency dependent tip-sample electrical interactions. Contrary to macroscopic methods, the high lateral resolution of nDS allows to study the dielectric properties of the polymer thin films at length scales of the order of the usual size of the amorphous and crystalline PEO domains $\left(10-10^{2} \mathrm{~nm}\right) \cdot{ }^{26-29}$ By means of nDS we were able to study to what extent the local dielectric function of PEO thin films can be laterally-resolved (40 $\mathrm{nm}$ resolution) and how it depends on film preparation conditions. The $\mathrm{nDS}$ signal was found to be independent of the specific tip position on the PEO thin film surface for three investigated samples. However, clear differences were observed depending on the PEO thin film preparation conditions. These findings were assigned to the strong effect of the preparation conditions on the resulting PEO structure and particularly on the amorphous phase characteristics. Based on a film dielectric function corresponding to the Maxwell/Wagner/Sillars (MWS) model, we calculated how the ionic conductivity distribution depends on the PEO thin film characteristics. 


\section{Experimental Methods}

A. Samples. Polyethylene oxide (PEO, Sigma-Aldrich, $\mathrm{Mw}=94000 \mathrm{Da}$, PDI $=1.18$, as determined by Gel Permeation Chromatography) thin films were prepared by spin coating polymer solutions (3600 rpm, $2 \mathrm{~min})$ using acetonitrile at $3 \mathrm{wt} \%\left(\mathrm{CH}_{3} \mathrm{CN}\right.$, anhydrous, Aldrich) and chloroform at $1 \mathrm{wt} \%\left(\mathrm{CHCl}_{3}\right.$, anhydrous, Aldrich). We have used electrically grounded gold-coated silicon wafers as substrates. Prior spin coating the solutions were left under stirring for $24 \mathrm{~h}$ in order to achieve good solubility. For both solvents, under these preparation conditions, we obtained PEO thin films with a thickness $(h)=200 \pm 10 \mathrm{~nm}$, as determined by Atomic Force Microscopy (AFM). From now on, we will denote the PEO films prepared from acetonitrile as $\mathrm{PEO}_{\mathrm{CH} 3 \mathrm{CN}}$, while the ones prepared from chloroform as $\mathrm{PEO}_{\mathrm{CHCl} 3}$. In order to remove the residual solvent, all thin films were left for, at least $24 \mathrm{~h}$, at room temperature under high vacuum (about $10^{-7}$ mbar). Poly(vinyl acetate) (PVAc, Mw $=240000 \mathrm{Da}$, Aldrich) was used as standard for the setup calibration. 200nm thick PVAc thin films were prepared in a similar way from a toluene solution at $3 \mathrm{wt} \%$.

B. nanoDielectric Spectroscopy (nDS). nDS measurements were performed using a Bruker Multimode 8 AFM, equipped with a Nanoscope V controller, under a high purity nitrogen flow. This non-contact technique allows to perform both the imaging of the sample's surface and the dielectric characterization of the material. The system also allows performing experiments above room temperature by using the Thermal Application Controller (TAC, Bruker).

In this work, we have used PtIr coated PFTUNA probes by Bruker. These probes have a nominal resonant frequency of $75 \mathrm{kHz}$ and an elastic constant of about $0.4 \mathrm{~N} / \mathrm{m}$. In $\mathrm{nDS}$, first we took images of the PEO topography using the conventional tapping mode, and then, at selected locations on the polymer surface, nDS data were recorded using a double-pass procedure. In the latter case, the mechanical excitation actuating on the probe was set to zero in order to keep a constant tip-sample distance, the probe was maintained above the surface at $z_{0}=$ $20 \mathrm{~nm}$, and a $3 \mathrm{~V}$ sinusoidal voltage with a frequency in the range $1 \leq f(\mathrm{~Hz}) \leq 50000$ was applied to the AFM probe, using a Stanford Research SR830 Lock-in amplifier (LIA), controlled via a homemade LabView routine. In the presence of an alternating electric field, the interaction between the probe and the polarizable entities in the PEO film resulted in a time dependent electrical force, which induced probe oscillations. We analyzed the component of this electrical force at a frequency double than that of the applied voltage. This component is directly connected with the probe-sample system characteristics as: ${ }^{22-25}$

$$
F^{*} \omega_{e}(t)=-\frac{1}{4} \frac{\partial C^{*}}{\partial z} V_{0}^{2} \cos \left(2 \omega_{e} t\right)
$$

where $C^{*}$ is the complex capacitance of the probe-sample system depending on the film dielectric function $\left(\varepsilon^{*}(\omega)=\varepsilon^{\prime}(\omega)-i \varepsilon^{\prime \prime}(\omega)\right), z$ is the coordinate along which the tip-sample distance is measured, $V_{0}$ is the amplitude of the AC voltage and $\omega_{e}=2 \pi f$. Then, the photodiode signal was analyzed by the LIA to obtain phase $(\theta)$ of the probe oscillations. The final nDS signal was obtained by calculating the frequency dependent phase shift $\Delta \theta(\omega)$, defined as:

$$
\Delta \theta(\omega)=\theta_{r e f}(\omega)-\theta(\omega)
$$


where $\theta_{\text {ref }}(\omega)$ is the probe oscillation phase associated with the electronics and the mechanical characteristics of the AFM probe, which has to be determined using a thin film with negligible electrical losses $\left(\varepsilon^{\prime \prime}(\omega)=0\right)$. In this work, $\theta_{\text {ref }}(\omega)$ was measured using a $200 \mathrm{~nm}$ PVAc thin film at room temperature.

C. nDS Analysis. To properly analyze the nDS signal, the system capacitance $C^{*}(\omega)$ needs to be modeled. As discussed by Miccio et al., ${ }^{25}$ the connection between the output signals of a nDS experiment and the material properties is not straightforward. In their work, authors developed a physical model of the effective capacitance between the AFM probe and a thin film sample by considering separately the contributions from the three parts of the probe: apex of the tip, cone, and cantilever. Since it was demonstrated that the contributions to the nDS signal arising from the cone can be neglected, in the present work we have taken into consideration exclusively the tip and cantilever contributions. Then, following the cited procedure, the tip contribution was modeled according to the works by Gomila et al., ${ }^{30-32}$ leading to:

$$
F_{2 \omega_{e}-T I P}^{*}(\omega) \propto 2 \pi \varepsilon_{0} \frac{R^{2}\left(1-\sin \Theta_{0}\right)}{z_{0}^{2}+z_{0} R\left(1-\sin \Theta_{0}\right)}\left(1+\frac{h}{z_{0} \varepsilon^{*}{ }_{L C}(\omega)}\right)^{-1}\left(1+\frac{h}{\left[z_{0}+R\left(1-\sin \Theta_{0}\right)\right] \varepsilon_{L C}^{*}(\omega)}\right)^{-1}
$$

where $\varepsilon_{0}=8.85 \times 10^{-12} \mathrm{~F} / \mathrm{m}$ is the vacuum permittivity, $R$ the effective tip radius and $\Theta_{0}$ the cone angle. The subscript $L C$ in $\varepsilon^{*}(\omega)$ denotes that, in general, this complex dielectric function will be related to the local properties of the material. On the other hand, the cantilever contribution can be written as: ${ }^{25}$

$$
F_{2 \omega_{e}-C A N T}^{*}(\omega) \propto \frac{\varepsilon_{0} W \xi}{T}\left[\left(1+\frac{h}{T \varepsilon^{*}{ }_{A V}(\omega)}\right)^{-1}-\frac{T}{L \sin \alpha} \ln \left(1+\frac{L \sin \alpha}{T}\left(1+\frac{h}{T \varepsilon^{*}{ }_{A V}(\omega)}\right)^{-1}\right)\right]
$$

where $W$ is the cantilever width, $L$ the cantilever length, $\alpha$ the cantilever angle, $T$ the cone length, and $\xi$ accounts for the border effects. The subscript $A V$ in $\varepsilon^{*}(\omega)$ denotes that it refers to an average film property, since this cantilever contribution is non-local. Combining equations (3) and (4) the probe-sample electrical force can be written as: $F^{*}{ }_{2 \omega_{e}}(\omega)=F^{*}{ }_{2 \omega_{e-} T I P}(\omega)+$ $F^{*}{ }_{2 \omega_{e}-C A N T}(\omega)$, and finally the phase shift measured by $\mathrm{nDS}$ (eq. 2 ) was calculated as:

$$
\Delta \theta(\omega)=\tan ^{-1}\left\{\frac{\operatorname{Im}\left[F^{*} 2 \omega_{e}(\omega)\right]}{\operatorname{Re}\left[F^{*}{ }_{2 \omega_{e}}(\omega)\right]}\right\}
$$

Note that this quantity is independent of the photodiode sensitivity and spring constant of the probe. However, it is worth highlighting that the magnitude of the nDS response will be dependent not only on the material properties, via $\varepsilon^{*}(\omega)$, but also on the geometrical characteristics of the AFM probe. Nevertheless, the frequency dependence of the phase shift is hardly affected by the cantilever contributions. For these reasons the values used for the geometrical characteristics of the probe other than the tip radius were those provided by the probe manufacturer. The effective tip radius, involved in the tip apex contribution, was 
determined for each particular probe by the calibration procedure described as follows. The AFM sample stage was heated up to $333 \mathrm{~K}$ and $\mathrm{nDS}$ measurements were carried out on a PVAc thin film (the same used in determining $\theta_{\text {ref }}(\omega)$ ). At this temperature the segmental dielectric relaxation of PVAc is clearly detectable within the experimental window of the nDS setup. ${ }^{22,24,}$ ${ }^{25}$ The resulting $\Delta \theta(\omega)$ signal was modeled following equations (3) - (5) and using for $\varepsilon^{*}(\omega)$ the Havriliak-Negami function with fixed parameters as described in refs. ${ }^{25,33,34}$. Finally, the value of $R$ in (3) that provided the most appropriate description of the PVAc data was established and used for the subsequent analysis of the nDS experiments on PEO films.

\section{Results}

Figure 1 (left column) shows nDS phase shifts $\Delta \theta$ as a function of the applied electric field frequency for three PEO thin films. For all samples the nDS signal shows up as a peak, which position and magnitude is sample-dependent. We recall that, since the geometrical characteristics of the probe play an important role on the nDS signal magnitude, it is not possible to compare directly the $\Delta \theta(\omega)$ values. For each sample, the different nDS data points were taken at the locations highlighted in their corresponding AFM topography images (Figure 1 , right column). It is possible to observe that, in every sample, the position of the $\mathrm{nDS}$ peak is only weakly dependent on the location on the sample surface where the $\mathrm{nDS}$ experiment was performed, i.e. the recorded signal is rather homogeneous. This is an indication that in all samples the relevant dielectric phenomena in the films occur within a volume with a lateral size below the typical resolution of our $\mathrm{nDS}$ experiment $(\sim 40 \mathrm{~nm})$.

Specifically, Figure 1a shows the results for the $200 \mathrm{~nm} \mathrm{PEO}_{\mathrm{CH} 3 \mathrm{CN}}$ thin film. In this case, the peaks maxima are located around $f=20 \mathrm{~Hz}$. The AFM topography images show that the surface of this film is completely covered by elongated nanostructures with varying lengths (from $10^{2}-$ $10^{3} \mathrm{~nm}$ ) and almost constant widths of $25 \pm 5 \mathrm{~nm}$. These features correlate nicely with the semicrystalline nature of the PEO and are fairly comparable to those previously reported in the literature for other PEO thin films. ${ }^{16,35-38}$ On the other hand, the results for the $200 \mathrm{~nm} \mathrm{PEO} \mathrm{CHCl}$ thin film are presented in Figure 1b. Here the maxima of the nDS peaks are located at around $f=$ $300 \mathrm{~Hz}$ and the signal is more extended towards low- $f$ values. Right side of Figure $1 \mathrm{~b}$ shows the corresponding AFM topography images. As in the previous case, the PEO film is formed by a superposition of nanometric features; however, the uniform distribution of elongated nanostructures observed in the $\mathrm{PEO}_{\mathrm{CH} 3 \mathrm{CN}}$ sample is lost. In $\mathrm{PEO}_{\mathrm{CHCl} 3}$ the features are much wider and longer, even showing flat areas on its surface. As already mentioned, the variations of the crystalline arrangement depending on the solvent used for the PEO films preparation have been recently investigated by Toolan et al. ${ }^{15}$ In their work, authors found that solvent polarity played an important role determining the final crystalline structure, in line to what we observed here via AFM. Moreover, our nDS data show that there is a dramatic change in the nDS signal depending on the solvent used, which could ultimately be related to changes in the arrangement of the polymer chains, particularly in the amorphous phase, as discussed in the following section. 

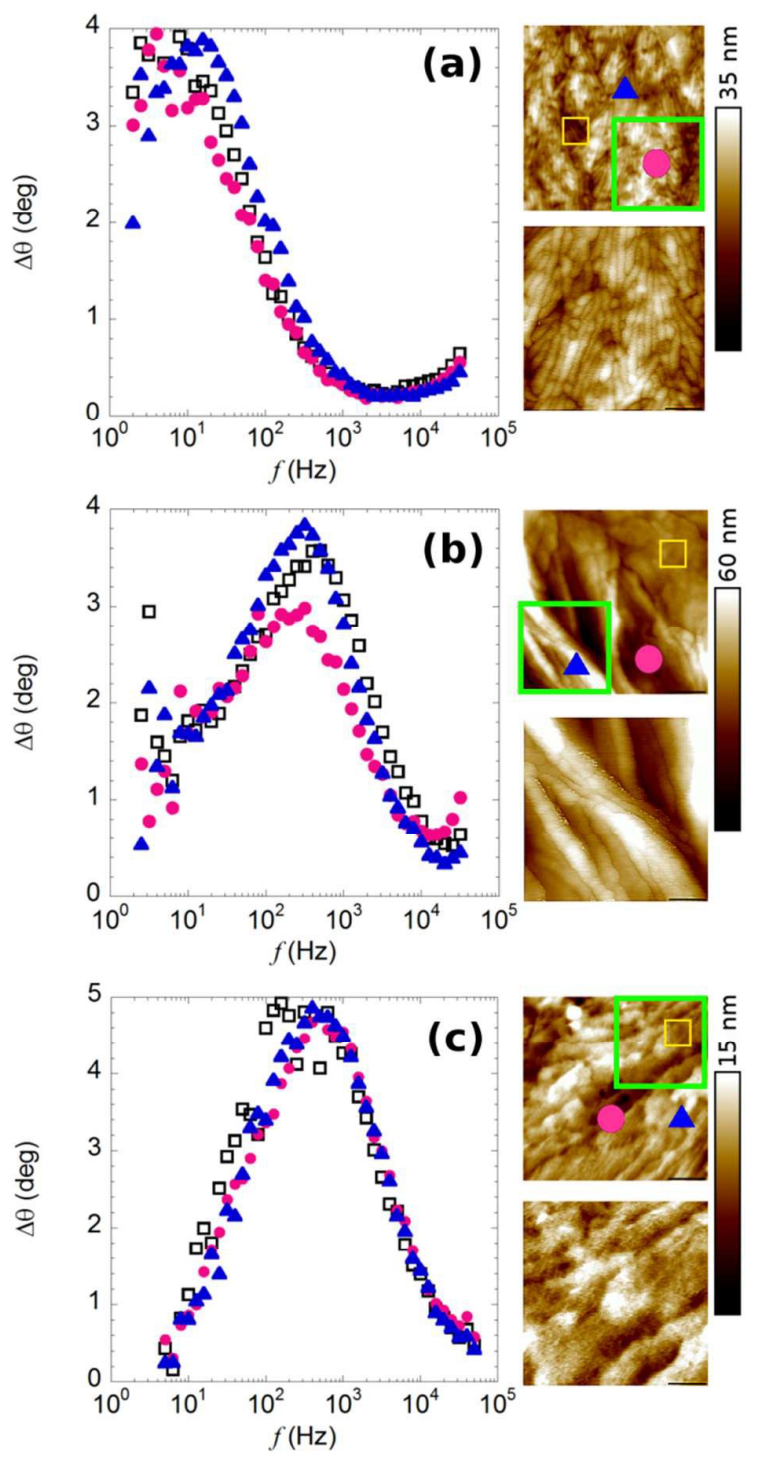

Figure 1. nDS signal (left column) and AFM topography images (right column) for different PEO thin films. (a) $200 \mathrm{~nm} \mathrm{PEO}_{\mathrm{CH} 3 \mathrm{CN}}$ thin film. (b) $200 \mathrm{~nm}$ PEO $_{\text {СHCl3 }}$ thin film. (c) $200 \mathrm{~nm}$ PEO $_{\text {melt }}$ thin film. All AFM images have lateral dimensions of $1 \mu \mathrm{m}$ (top) and $500 \mathrm{~nm}$ (bottom), being the latter a zoom into the area highlighted with a green square.

In order to cut out the solvent dependence, the PEO thin films were melted and recrystallized inside the AFM stage. For this procedure, maintaining a continuous $\mathrm{N}_{2}$ flow, the sample stage was heated up to $373 \mathrm{~K}$ and this temperature was hold for $15 \mathrm{~min}$. Afterwards, the sample was allowed to cool down to $298 \mathrm{~K}$. The nDS results after the thermal treatment are shown in Figure $1 \mathrm{c}$, and from now on we will denote this sample as $\mathrm{PEO}_{\text {melt }}$. The $\mathrm{nDS}$ spectra show symmetric peaks, centered about $f=500 \mathrm{~Hz}$. This position of peak maximum is comparable to that found for the $\mathrm{CHCl}_{3}$, although the shape of the nDS peak is significantly altered. The fact that $f_{\max }($ melt $) \approx f_{\max }\left(\mathrm{CHCl}_{3}\right)>f_{\max }\left(\mathrm{CH}_{3} \mathrm{CN}\right)$ goes in line with the results by Toolan and collaborators, ${ }^{15}$ showing that the thin films formed from low-polar solvents have a similar chain arrangement to those formed from the melt, whereas those formed from highly polar solvents are markedly different. ${ }^{15}$ Finally, it is noteworthy that the AFM topography images of $\mathrm{PEO}_{\text {melt }}$ show a smoother surface (refer to the vertical bar to the right of the images), comprised by nanostructured material but without the clear definition found in the as-casted samples. 
In Figure 2 we present the mean values of $\Delta \theta(\omega)$ for the investigated samples (open symbols). Since the nDS signal was nearly independent on the tip location, the data points for each PEO film were obtained as the mathematical average of the 3 curves shown in Figure 1 and would represent the average film properties. Also, in each panel we have included the values of the most relevant AFM probe parameters, those that could influence the magnitude of $\Delta \theta$, particularly, the cantilever area, the cone height (both provided by the probe manufacturer), and the effective tip radius as determined via the calibration experiment using PVAc.
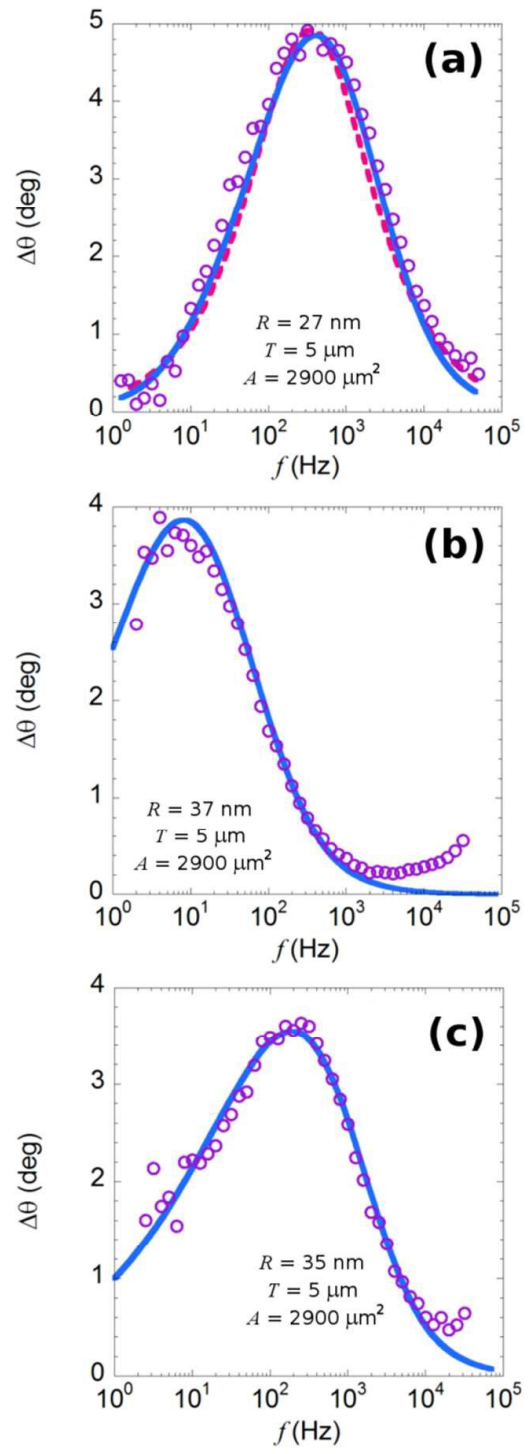

Figure 2. Average nDS signal (symbols) and fittings (lines) for (a) $\mathrm{PEO}_{\text {melt }}$, (b) $\mathrm{PEO}_{\mathrm{CHCl} 3}$. (c) $\mathrm{PEO}_{\mathrm{CH} 3 \mathrm{CN}}$. For each panel, $R$ represents the tip radius, $T$ the cone height and $A$ the cantilever area $(A=W \cdot L)$. 


\section{Discussion}

Taking into consideration the semicrystalline nature of the PEO, as well as recent reports on its molecular dynamics that evidenced extremely fast segmental motions at room temperature, ${ }^{39}$ the detected nDS signal must be related to Maxwell/Wagner/Sillars (MWS) polarization phenomena originated by the blocking of charges at the internal phase boundaries. ${ }^{40}$ The welldefined and rather symmetrical shape of the $\mathrm{nDS}$ data corresponding to the $\mathrm{PEO}_{\text {melt }}$ sample (Figure 2a), led us to choose this sample to begin a detailed data analysis. As an starting point we have assumed that the dielectric relaxation function of this film could be well described by a Cole-Cole $(\mathrm{CC})$ dielectric function $\left(\varepsilon_{\mathrm{CC}}\right)^{40}$

$$
\varepsilon^{*}{ }_{C C}(\omega)=\varepsilon_{\infty}+\frac{\Delta \varepsilon}{\left(1+i \omega / \omega_{\max }\right)^{b}}
$$

where $\varepsilon_{\infty}=\lim _{\omega \rightarrow \infty} \varepsilon^{\prime}(\omega)$ relates to the fast polarization mechanisms, $\Delta \varepsilon$ is the relaxation strength and $\omega_{\max }$ the peak frequency of the dielectric loss curve. The parameter $b(0<b \leq 1)$ relates to the symmetric broadness of the dielectric loss curve. In the limiting case of $b=1$ eq. (6) corresponds to the Debye equation. ${ }^{40}$ Since the nDS signal essentially represents the film properties in any location of the film surface, in equations (3) and (4) it holds that $\varepsilon_{L C}^{*}=\varepsilon^{*}{ }_{A V}=$

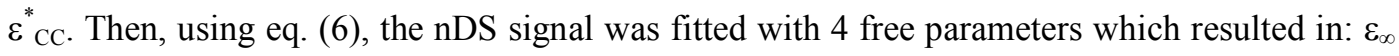
$=3.1 \pm 0.2, \Delta \varepsilon=11 \pm 1, \omega_{\max }=1.3 \times 10^{3} \mathrm{~s}^{-1}( \pm 25 \%)$ and $b=0.70 \pm 0.02$. The corresponding fitting curve is shown in Figure $2 \mathrm{a}$ by the dashed line, where a good agreement can be observed.

Taking into account the non-local character of the nDS signal from the PEO films, the CC description of the dielectric function of the $\mathrm{PEO}_{\text {melt }}$ should account for the MWS interfacial polarization process at the amorphous/crystalline interfaces. These interfaces most probably would be those present in the lamellar stacking typical of semicrystalline polymers as PEO. The large period of the lamellar structure of bulk PEO is about $20 \mathrm{~nm},{ }^{41}$ which would be compatible with fact that the measured nDS signal is essentially the same, independently of the tip location on the film surface. A semicrystalline polymer can be viewed as a mixture of the nonconducting crystalline phase, occupying a volume fraction $\varphi$ and having frequency independent dielectric constant $\varepsilon_{c}$, and the amorphous phase having conductivity $\sigma$ and dielectric constant $\varepsilon_{a}$. For PEO films at room temperature $\varepsilon_{a}$ can be also taken as frequency independent. When a layered structure is assumed the resulting dielectric function is given by the MWS expression: ${ }^{40}$

$$
\varepsilon^{*}{ }_{\mathrm{MWS}}(\omega)=\varepsilon_{\infty_{-} \mathrm{MWS}}+\frac{\Delta \varepsilon_{\mathrm{MWS}}}{1+i \omega / \omega_{\mathrm{MWS}}}
$$

where the parameters are related to the two components properties by:

$$
\begin{gathered}
\varepsilon_{\infty_{-} \mathrm{MWS}}=\frac{\varepsilon_{c} \varepsilon_{a}}{\varepsilon_{c}(1-\varphi)+\varepsilon_{a} \varphi} \\
\Delta \varepsilon_{\mathrm{MWS}}=\frac{\varepsilon_{c}}{\varphi}\left[1-\frac{\varepsilon_{a}}{\frac{\varepsilon_{c}}{\varphi}(1-\varphi)+\varepsilon_{a}}\right]
\end{gathered}
$$




$$
\omega_{\mathrm{MWS}}=\frac{\sigma \varphi}{\varepsilon_{0}\left[\varepsilon_{c}(1-\varphi)+\varepsilon_{a} \varphi\right]}
$$

Under this approach, the position of the dielectric loss peak maxima $\left(\omega_{\mathrm{MwS}}\right)$ will be essentially dictated by conductivity of the amorphous phase $\sigma$, while the intensity of the peak ( $\Delta \varepsilon_{\mathrm{MwS}}$ ) will mostly depend on $\varphi$.

The CC and the MWS expressions are equivalent only when the shape parameter $b$ takes the value 1 (Debye relaxation). However, the fitting of the PEO film nDS data required a significantly lower value of $b=0.7$. Thus, the application of the MWS model to our data is not direct. Values of $b<1$ in the CC equation can be interpreted as the result of the superposition of distinct Debye contributions, each with a different characteristic frequency. ${ }^{40}$ In this framework, the dielectric function of the semicrystalline polymer could be expressed as:

$$
\varepsilon^{*}(\omega)=\int_{0}^{\infty} \varepsilon^{*}{ }_{M W S}(\omega) g\left(\log _{10} \sigma\right) \mathrm{d}\left(\log _{10} \sigma\right)
$$

where $\varepsilon^{*}{ }_{\text {MwS }}(\omega)$ is given by eq. (7) and $g(\log \sigma)$ corresponds to a distribution function of conductivities in the amorphous phase. The presence of a distribution of conductivities would be expected by considering the ill-defined nature of the amorphous phase in semicrystalline polymers ${ }^{11,42}$ that can range from the most conventional fully amorphous state of a polymer melt, to the so called rigid amorphous phase where segmental mobility is fully impeded. ${ }^{12}$ In general, the experimental results suggest a gradual variation of the characteristics of the amorphous phase on approaching the crystalline phase. ${ }^{42}$

With these ideas in mind, the nDS data in Figure 2a has been refitted using eq. (5) in combination with eq. (9). The permittivity values of the crystal and amorphous phases where taken as $\varepsilon_{c}=2.5$ and $\varepsilon_{a}=3.0$, respectively. In the fitting, $g(\log \sigma)$ was assumed to be a Gaussian-like function and therefore we let 3 fitting parameters free. A very good agreement between the model and the data points was found. A volume fraction $\varphi=0.18$ accounts for the nDS signal amplitude whereas the corresponding distribution function $g(\log \sigma)$ is shown in Figure 3 (solid line). According to this description the conductivity in the amorphous phase of the $\mathrm{PEO}_{\text {melt }}$ film ranges from about $10^{-7}$ to $10^{-11} \mathrm{~S} / \mathrm{cm}$, i.e. about 4 orders of magnitude, with a peak value $1.710^{-9} \mathrm{~S} / \mathrm{cm}$. It is noteworthy that the higher conductivity values of around $10^{-7} \mathrm{~S} / \mathrm{cm}$ nearly corresponds to the room temperature DC conductivity of fully amorphous PEO, as determined by extrapolating from the bulk melt state behavior. On the other hand, the 4 decades conductivity gradient obtained goes in line with the typical gradient of the amorphous segmental mobility usually found in semicrystalline polymers. ${ }^{10}$ 


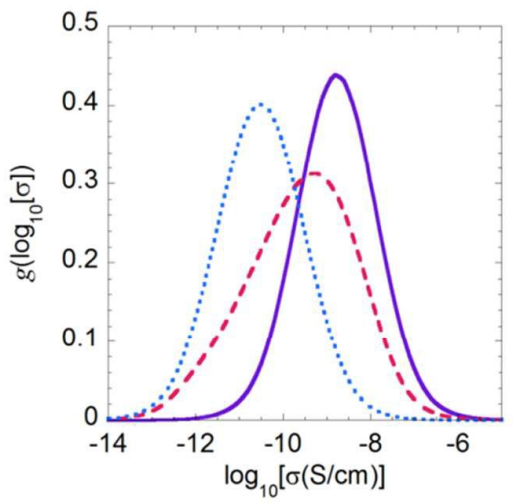

Figure 3. Distribution function of the matrix conductivity for $\mathrm{PEO}_{\mathrm{CH} 3 \mathrm{CN}}$ (dotted line), $\mathrm{PEO}_{\mathrm{CHCl3}}$ (dashed line) and $\mathrm{PEO}_{\text {melt }}$ (solid line).

Taking these results into account, the nDS data obtained for $\mathrm{PEO}_{\mathrm{CH} 3 \mathrm{CN}}$ and $\mathrm{PEO}_{\mathrm{CHCl} 13}$ films were fitted using the same approach (Figures $2 \mathrm{~b}$ and $2 \mathrm{c}$, respectively). Concerning the value of the volume fraction $\varphi$, we found that maintaining $\varphi=0.18$ allows a good fitting, provided that the conductivity distribution function $g(\log \sigma)$ is properly modified. Particularly, a Gaussian-like distribution with a peak value of $3.410^{-11} \mathrm{~S} / \mathrm{cm}$ extending over about 5 decades is found to describe well the data for $\mathrm{PEO}_{\mathrm{CH} 3 \mathrm{CN}}$ (see Figure 3, dotted line), whereas the conductivity distribution becomes asymmetric for $\mathrm{PEO}_{\mathrm{CHCl}}$ with a peak value of $5.610^{-10} \mathrm{~S} / \mathrm{cm}$ and conductivity values ranging from $10^{-7}$ to $10^{-13} \mathrm{~S} / \mathrm{cm}$ (6 decades, as shown by the dashed line in Figure 3). These results demonstrate that there are major differences in the PEO amorphous phase arrangements resulting from the different film characteristics. The $\mathrm{PEO}_{\text {melt }}$ film shows the narrower distribution, which can be interpreted due to the presence of a more homogeneous amorphous phase. Moreover it also shows the highest average conductivity value that would mean a less efficient packing in the amorphous phase. Opposite, the PEO film prepared from a highly polar solvent solution $\left(\mathrm{PEO}_{\mathrm{CH} 3 \mathrm{CN}}\right)$ presents a very low average conductivity that would be indicative of a more efficient packing of its amorphous phase. The case of $\mathrm{PEO}_{\mathrm{CHC} 13}$ represents an intermediate situation where it seems that both highly packed and loosely packed polymer strands would coexist in the amorphous phase of this film.

Our findings are coherent with the reported results on the influence of the preparation conditions on the crystalline structure of PEO thin films. ${ }^{15}$ However, here we detect changes occurring in the amorphous phases that should be associated to corresponding changes in the PEO crystallization. For $\mathrm{PEO}_{\mathrm{CH} 3 \mathrm{CN}}$ we found a rather packed amorphous phase, which is in agreement with the suggested idea that in this film there are cluster-type structures that difficult the chain-straightening formation giving rise to the lamella structure. ${ }^{15}$ On the other hand, despite the fact that the reported differences between $\mathrm{PEO}_{\mathrm{CHCl}}$ and $\mathrm{PEO}_{\text {melt }}$ films concerning crystallization are found to be minor, ${ }^{15}$ our experiments put in evidence important differences in the characteristics of the respective amorphous phases. Noteworthy, the detected changes in the amorphous phase of the PEO films, prepared by different methods, will impact not only the ionic transport but also other technologically relevant properties as gas permeability, for instance. 


\section{Conclusions}

In the present work we used nDS to analyze the ionic transport in $200 \mathrm{~nm}$ PEO thin films prepared by three different methods. In all the films we found that the nDS signal is essentially independent of the AFM tip location on the film surface and, consequently, has to be assigned to interfacial polarization phenomena occurring at the crystal/amorphous interfaces of PEO lamellar structures. Our experiments evidenced profound changes in the characteristics of the amorphous phase of the PEO, a result in line with the reported effect of the PEO thin film preparation conditions on the resulting crystalline structures. Particularly, we found that PEO films prepared by spin coating from solutions in a highly polar solvent present an amorphous phase with relatively low conductivity $\left(\sim 310^{-11} \mathrm{~S} / \mathrm{cm}\right)$, indicating efficient molecular packing. Contrary, when the same film is melted and crystallized from the melt, the average conductivity of the resulting amorphous phase increases dramatically, around two orders of magnitude, signifying poorer molecular packing. In this case, the conductivity values in the amorphous phase are found to be distributed according to a log-Gaussian function with a high-conductivity tail reaching the value expected at room temperature for fully amorphous PEO $\left(\sim 10^{-6} \mathrm{~S} / \mathrm{cm}\right)$. The films prepared by spin coating from a PEO solution in a low-polar solvent present an intermediate situation. In summary, the changes in conductivity of the amorphous phases evidence the profound effect of the preparation method of the PEO films on the ionic transport.

\section{Acknowledgements}

The authors acknowledge the Basque Country Government (Reference No. IT-654-13 (GV)), and Spanish Government (Grant No. MAT2015-63704-P, (MINECO/FEDER, UE)) for their financial support.

\section{References}

1. Z. Gadjourova, Y. G. Andreev, D. P. Tunstall and P. G. Bruce, Nature, 2001, 412, 520-523.

2. M. Patel, P. V. Menezes and A. J. Bhattacharyya, The Journal of Physical Chemistry B, 2010, 114, 5233-5240.

3. Z. Xue, D. He and X. Xie, Journal of Materials Chemistry A, 2015, 3, 19218-19253.

4. A. Manthiram, X. Yu and S. Wang, Nature Reviews Materials, 2017, 2, 16103.

5. J. M. Tarascon and M. Armand, Nature, 2001, 414, 359-367.

6. M.-C. Yang, H.-W. Cho and J.-J. Wu, Nanoscale, 2014, 6, 9541-9544.

7. M. Kammoun, S. Berg and H. Ardebili, Nanoscale, 2015, 7, 17516-17522.

8. S. Cheng, D. M. Smith and C. Y. Li, Macromolecules, 2014, 47, 3978-3986.

9. A. M. Christie, S. J. Lilley, E. Staunton, Y. G. Andreev and P. G. Bruce, Nature, 2005, 433, 50-53.

10. A. Alegria and J. Colmenero, Soft Matter, 2016, 12, 7709-7725.

11. I. Arandia, A. Mugica, M. Zubitur, R. Mincheva, P. Dubois, A. J. Müller and A. Alegría, Macromolecules, 2017, DOI: 10.1021/acs.macromol.6b02713.

12. C. Schick, A. Wurm and A. Mohammed, in Polymer Crystallization: Observations, Concepts and Interpretations, eds. G. Reiter and J.-U. Sommer, Germany, 2003.

13. X. Shen, W. Hu and T. P. Russell, Macromolecules, 2016, 49, 4501-4509.

14. H.-G. Braun and E. Meyer, International Journal of Molecular Sciences, 2013, 14, 3254.

15. D. T. W. Toolan, A. Isakova, R. Hodgkinson, N. Reeves-McLaren, O. S. Hammond, K. J. Edler, W. H. Briscoe, T. Arnold, T. Gough, P. D. Topham and J. R. Howse, Macromolecules, 2016, 49, 4579-4586. 16. M. Asada, N. Jiang, L. Sendogdular, J. Sokolov, M. K. Endoh, T. Koga, M. Fukuto, L. Yang, B. Akgun, M. Dimitriou and S. Satija, Soft Matter, 2014, 10, 6392-6403.

17. D. E. Martínez-Tong, B. Vanroy, M. Wübbenhorst, A. Nogales and S. Napolitano, Macromolecules, 2014, 47, 2354-2360.

18. S. Napolitano, S. Capponi and B. Vanroy, The European Physical Journal E, 2013, 36.

19. W. Zeng, L. Shu, Q. Li, S. Chen, F. Wang and X.-M. Tao, Advanced Materials, 2014, 26, 5310-5336. 
20. Z. Tehrani, T. Korochkina, S. Govindarajan, D. J. Thomas, J. O’Mahony, J. Kettle, T. C. Claypole and D. T. Gethin, Organic Electronics, 2015, 26, 386-394.

21. L. Wang, Y. Zhang, J. Pan and H. Peng, Journal of Materials Chemistry A, 2016, 4, 13419-13424.

22. G. A. Schwartz, C. Riedel, R. Arinero, P. Tordjeman, A. Alegría and J. Colmenero, Ultramicroscopy, 2011, 111, 1366-1369.

23. M. M. Kummali, A. Alegría, L. A. Miccio and J. Colmenero, Macromolecules, 2013, 46, 7502-7512.

24. L. A. Miccio, M. M. Kummali, G. A. Schwartz, Á. Alegría and J. Colmenero, Ultramicroscopy, 2014, 146, 55-61.

25. L. A. Miccio, M. M. Kummali, G. A. Schwartz, Á. Alegría and J. Colmenero, Journal of Applied Physics, 2014, 115, 184305.

26. A. J. Bhattacharyya, J. Fleig, Y. G. Guo and J. Maier, Advanced Materials, 2005, 17, 2630-2634.

27. W. Lee, F. B. Prinz, X. Chen, S. Nonnenmann, D. A. Bonnell and R. P. O'Hayre, MRS Bulletin, 2012, 37, 659-667.

28. Y.-H. Liu, S.-S. Liao and B. Haochih Liu, Nanoscale, 2016, 8, 19978-19983.

29. J. Kruempelmann, M. Balabajew, M. Gellert and B. Roling, Solid State Ionics, 2011, 198, 16-21.

30. G. Gomila, J. Toset and L. Fumagalli, Journal of Applied Physics, 2008, 104, 024315.

31. L. Fumagalli, G. Ferrari, M. Sampietro and G. Gomila, Nano Letters, 2009, 9, 1604-1608.

32. L. Fumagalli, G. Gramse, D. Esteban-Ferrer, M. A. Edwards and G. Gomila, Applied Physics Letters, 2010, 96, 183107.

33. G. A. Schwartz, E. Tellechea, J. Colmenero and Á. Alegría, Journal of Non-Crystalline Solids, 2005, 351, 2616-2621.

34. M. Tyagi, A. Alegría and J. Colmenero, The Journal of Chemical Physics, 2005, 122, 244909.

35. G. Reiter and J.-U. Sommer, Physical Review Letters, 1998, 80, 3771-3774.

36. J. K. Hobbs, C. Vasilev and A. D. L. Humphris, Polymer, 2005, 46, 10226-10236.

37. G.-1. Zhang, L.-x. Jin, P. Zheng, W. Wang and X.-j. Wen, Chinese Journal of Polymer Science, 2013, 31, 798-808.

38. J. Zhao, X. Yin, J. Shi, X. Zhao and J. S. Gutmann, The Journal of Physical Chemistry C, 2011, 115, 22347-22353.

39. C. Do, P. Lunkenheimer, D. Diddens, M. Götz, M. Weiß, A. Loidl, X.-G. Sun, J. Allgaier and M. Ohl, Physical Review Letters, 2013, 111, 018301.

40. F. Kremer and A. Schönhals, Broadband Dielectric Spectroscopy, Springer, 2003.

41. W. H. de Jeu, Basic X-Ray Scattering for Soft Matter, Oxford University Press, 2016.

42. A. Esposito, N. Delpouve, V. Causin, A. Dhotel, L. Delbreilh and E. Dargent, Macromolecules, 2016, 49, 4850-4861. 


\title{
Table of Contents Entry
}

\section{Ionic transport in the amorphous phase of semicrystalline polyethylene oxide thin films}

\author{
Daniel E. Martínez-Tong ${ }^{1,2}$, Luis A. Miccio ${ }^{2, \dagger}$, Angel Alegria ${ }^{2,3}$ \\ ${ }^{1}$ Donostia International Physics Center. P. M. Lardizabal 4, 20018 Donostia, Spain. \\ ${ }^{2}$ Centro de Física de Materiales (CSIC-UPV/EHU). P. M. Lardizabal 5, 20018 Donostia, Spain. \\ ${ }^{3}$ Departamento de Física de Materiales (UPV/EHU), Apto. 1072, 20080 Donostia, Spain. \\ 'Present address: Bihurcrystal. P Mikeletegi 83, 20009, Donostia, Spain.
}

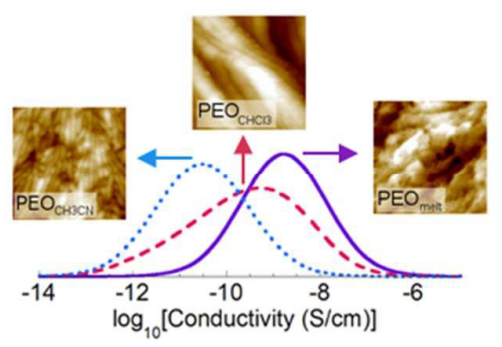

